\title{
Direct Relativistic Extension of the Madelung-de- Broglie-Bohm Reformulations of Quantum Mechanics and Quantum Hydrodynamics
}

\section{Arquimedes Ruiz-Columbié}

Texas Tech University

Luis Grave de Peralta ( $\square$ luis.grave-de-peralta@ttu.edu )

Texas Tech University

\section{Research Article}

Keywords: particle, mass, spin-0, relativistic relation, momentum , kinetic energy

Posted Date: February 8th, 2021

DOI: https://doi.org/10.21203/rs.3.rs-156006/v1

License: (c) (1) This work is licensed under a Creative Commons Attribution 4.0 International License.

Read Full License

Version of Record: A version of this preprint was published at Journal of Modern Physics on August 15th, 2021. See the published version at https://doi.org/10.4236/jmp.2021.1210085. 


\title{
Direct relativistic extension of the Madelung-de-Broglie-Bohm reformulations of quantum mechanics and quantum hydrodynamics
}

\author{
Arquimedes Ruiz-Columbié ${ }^{1}$ and Luis Grave de Peralta, ${ }^{2,3, *}$ \\ ${ }^{1}$ Wind Energy Program, Texas Tech University, Lubbock, TX 79409, USA \\ ${ }^{2}$ Department of Physics and Astronomy, Texas Tech University, Lubbock, TX 79409, USA \\ ${ }^{3}$ Nano Tech Center, Texas Tech University, Lubbock, TX 79409, USA \\ Email: luis.grave-de-peralta@ttu.edu
}

\begin{abstract}
Using a Schrödinger-like equation, which describes a particle with mass and spin-0 and with the correct relativistic relation between its linear momentum and kinetic energy, the basic equations of the non-relativistic quantum mechanics with trajectories and quantum hydrodynamics are extended to the relativistic domain. Some simple but instructive free particle examples are discussed.
\end{abstract}

\section{Introduction}

In 1927, shortly after E. Schrödinger published a seminal paper containing his celebrated equation [1], E. Madelung dared an interpretation showing that the Schrödinger equation can be transformed into two equations that mimic the continuity and the Euler equations of hydrodynamics [2]. The Euler equation is a particular case of the Navier-Stokes equation [3]. Such hydrodynamic interpretation is now considered a forebear of the de Broglie-Bohm Pilot Wave Theory [4-7], although germs of this theory were ventured in 1924 by L. de Broglie [8]. The process followed by Madelung consisted in expressing the Schrödinger solution in an exponential form which led to the two abovementioned equations, one for the amplitude and another for the phase. Those ideas were later retaken by D. Bohm [4-5]. Consequently, must of the work related to the Madelung-de-Broglie-Bohm reformulation of quantum mechanics and quantum hydrodynamics applies to particles moving slowly respect to the speed of light. A fully relativistic quantum mechanics with trajectories was recently formulated [9]; however, it lacks the relative simplicity of the non-relativistic formulation. Other general approaches have been reported [10], but we explore in this work an alternative methodology for extending, to the relativistic domain, the known non-relativistic quantum hydrodynamics and quantum theories with trajectories. Our approach is based in a surprising wave equation which resembles the Schrödinger equation, but describes a particle with mass and spin- 0 which moves through a potential $V$, and has the correct relativistic relation between the linear momentum $p$ and the kinetic energy $K$ [11-15]:

$$
i \hbar \frac{\partial}{\partial t} \psi(x, t)=-\frac{\hbar^{2}}{\left(\gamma_{v}+1\right) m} \frac{\partial^{2}}{\partial x^{2}} \psi(x, t)+V(x) \psi(x, t) .
$$

In Eq. (1), which is the GdeP equation for a particle with mass $m, \hbar$ is the Plank constant $(h)$ divided by $2 \pi$, and $\gamma_{\mathrm{v}}$ is a factor commonly found in special theory of relativity formulas (the Lorentz factor), which depends on the ratio between the squares of the particle's speed $\left(\mathrm{v}^{2}\right)$ and the speed of the light in the vacuum $\left(c^{2}\right)$ [16]: 


$$
\gamma_{\mathrm{v}}=\frac{1}{\sqrt{1-\frac{\mathrm{v}^{2}}{c^{2}}}}
$$

The basic properties of Eq. (1) and its solutions, and detailed discussions of how to solve Eq. (1) for some interesting potentials $V$, can be found in recently published works [11-15]. In a nutshell, solving Eq. (1) requires simultaneously finding the wavefunction $\psi$ and the square of the particle $v^{2}$, which determines the value of $\gamma_{v}$ in Eq. (2). This may look at first as an unmanageable problem; however, this is not the case in at least several interesting cases [11-15]. In general, Eq. (1) is non-lineal; this has been discussed before [11, 15]. Nevertheless, due the formal similitude with the Schrödinger equation, Eq. (1) is a useful and tractable equation. It is worth noting that Eq. (1) can be rewritten in the following way $[11,13,15]$ :

$$
i \hbar \frac{\partial}{\partial t} \psi=\widehat{H} \psi(x, t), \widehat{H}=\widehat{K}+V, \widehat{K}=\frac{\hat{p}^{2}}{\left(\gamma_{\mathrm{v}}+1\right) m}, \hat{p}=i \hbar \frac{\partial}{\partial x} .
$$

The operator $K$ corresponds to the exact relativistic kinetic energy of the particle, thus [11, $13,15]$ :

$$
\widehat{K}=\frac{\hat{p}^{2}}{\left(\gamma_{\mathrm{v}}+1\right) m}=\sqrt{\hat{p}^{2} c^{2}+m^{2} c^{4}}-m c^{2} .
$$

This means that Eq. (1) is well-defined, but it is advantageous to write the operator $K$ as in Eq. (1), because this results in an equation formally like the Schrödinger equation, which can then be exactly solved following similar procedures than the ones required for solving the Schrödinger equation [11-15]. It is also the striking similarity between Eq. (1) and the Schrödinger equation what allowed us to extend, to the relativistic domain, the basic equations of the Madelung-de-Broglie-Bohm reformulation of quantum mechanics and quantum hydrodynamics. It is worth noting that previous reports discussed how Eq. (1) with $V \equiv 0$ can be obtained from the Klein-Gordon equation for a free particle [13, 15]. Eq. (1) could be then understood as an eigenvalue equation where the eigenfunction $\psi$ and the eigenvalue $\gamma_{\mathrm{v}}$ should be simultaneously found [15]. From a pragmatic point of view, a Schrödinger-like equation appears to be very useful since Schrödinger-like solutions may apply. Investigating exact solutions using such an analogy might make more tractable some relativistic problems. In this work, we applied a methodology that extends already studied applications of the Schrödinger equation to the relativistic domain. This approach might become beneficial. From an epistemological point of view, the Schrödinger-like approach explored here should be considered as a "mathematical hypothesis" and the practical results must be examined as its final test. We will assume in this work this procedural interpretation of Eq. (1). A comprehensive discussion of the fundamental interpretation of Eq. (1) will published elsewhere. We hope that the scientific community, which is currently working on non-relativistic quantum mechanics theories with trajectories and quantum hydrodynamics, will recognize the simplicity of the theory presented in this work, and its potential for practical applications in relativistic quantum simulations. The rest of this work is organized in the following way. In the next Section, a relativistic extension of the de Broglie-Bohm quantum mechanics is obtained from the relativistic but Schrödinger-like GdeP equation. Then, a relativistic extension of the Madelung quantum electrodynamics is presented. This is followed by four free particle examples increasing in order of complexity. Finally, the conclusions of this work are given in the Conclusions. 


\section{Madelung-Bohm-like reformulation of the GdeP Equation}

The three-dimensional (3D) GdeP equation for a particle moving at relativistic speeds in a potential $V$ is given by the following expression [13-15]:

$$
i \hbar \frac{\partial}{\partial t} \psi=-\frac{\hbar^{2}}{\left(\gamma_{v}+1\right) m} \nabla^{2} \psi+V \psi \text {. }
$$

In general, the wavefunction $(\psi)$, the potential, and $\gamma_{\mathrm{v}}$ all depend on the three spatial coordinates and the time. Due to the formal similarity between Eq. (5) and the Schrödinger equation, a Madelung-Bohm-like extension of Eq. (5) can be done following the same procedure commonly used for reformulating the Schrödinger equation [2, 4-7]. First, we look for a solution of the GdeP equation of the following form:

$$
\psi(\vec{r}, t)=R(\vec{r}, t) e^{i S(\vec{r}, t) / \hbar} .
$$

In Eq. (4), $R$ and $S$ are the amplitude and phase fields, respectively [2, 4-7]. Inserting Eq [6] in Eq. (5) and following step by step Ref. [6], we can obtain the following equations, which extend to the relativistic domain the basic equations of the Madelung-de Broglie-Bohm quantum mechanics [6]:

$$
\begin{aligned}
& \frac{\partial}{\partial t} S+\frac{\nabla S^{2}}{\left(\gamma_{\mathrm{v}}+1\right) m}+[V+Q]=0, Q=-\frac{\hbar^{2}}{\left(\gamma_{\mathrm{v}}+1\right) m} \frac{\nabla^{2} R}{R} . \\
& \frac{\partial}{\partial t} R^{2}+\frac{2}{\left(\gamma_{\mathrm{v}}+1\right)} \nabla \cdot\left[\gamma_{\mathrm{v}} R^{2}\left(\frac{\nabla S}{\gamma_{\mathrm{v}} m}\right)\right]=0 .
\end{aligned}
$$

In Eq (7), $Q$ is the quantum potential [6]. Clearly, $\gamma_{\mathrm{v}} \approx 1$ when $\mathrm{v}^{2}<<c^{2}$; therefore, as it should be expected when the particle moves at low speeds, Eqs. (7) and (8) coincide to the well-known equations of the Madelung-de Broglie-Bohm quantum mechanics [6]. At relativistic velocities, the velocity field should now be defined such that the relation between the velocity and the linear momentum $(\nabla S)$ is the correct relativistic relationship [16]:

$$
\gamma_{\mathrm{v}} \overrightarrow{\mathrm{v}}=\frac{\nabla S}{m} \Rightarrow \overrightarrow{\mathrm{v}}=\frac{1}{\gamma_{\mathrm{v}}} \frac{\nabla S}{m} \text {. }
$$

Thus, the expression between parentheses in Eq. (8) is the velocity field given by Eq. (9). Again, when $v^{2} \ll<c^{2}$, Eq. (9) coincides with the non-relativistic equation [6]. However, in general:

$$
\overrightarrow{\mathrm{v}}=\frac{c}{\sqrt{(m c)^{2}+\nabla S^{2}}} \nabla S \Rightarrow \gamma_{\mathrm{v}}=\frac{\sqrt{(m c)^{2}+\nabla S^{2}}}{m c} .
$$

Therefore, when $\psi$ is known, Eq. (10) determines the velocity field and $\gamma_{\mathrm{v}}$. The velocity is then locally proportional to $\nabla S$ and its direction is perpendicular to the surfaces of constant phase $(S=$ constant). Bohm introduced a particle's trajectory as the solution of the following differential equation and initial conditions [4-7]:

$$
\frac{\partial}{\partial t} \vec{r}_{p}(t)=\overrightarrow{\mathrm{v}}\left(\vec{r}=\vec{r}_{p}(t), \mathrm{t}\right), \vec{r}_{p}(t=0)=\vec{r}_{o}
$$


Therefore, different trajectories correspond to different initial positions of the particle. The direction of the particle's velocity is always tangent to the particle's trajectory. The particle's velocity is given by the following equation:

$$
\overrightarrow{\mathrm{v}}_{p}(\mathrm{t})=\frac{\partial}{\partial t} \vec{r}_{p}(t)
$$

Equations (11) and (12) related the velocity of the Bohmian particle with the velocity of the Madelung's fluid.

\section{Relativistic quantum hydrodynamics}

Madelung did not introduce particle trajectories in his reformulation of the Schrödinger equation [2]. This was done later by Bohm [4-5]. Madelung interpreted Eqs. (7) and (8) as describing a fluid with density $\rho^{\prime}=m \rho$ such that:

$$
\rho(\vec{r}, t)=R^{2}(\vec{r}, t) \text {. }
$$

Then using Eq. (9) with $\gamma_{v}=1$ allowed him to directly rewrite Eq. (8) with $\gamma_{v}=1$ as a continuity equation [2, 6-7]. Proceeding in a similar way, we obtained the following extension of the Madelung's continuity equation to the relativistic domain:

$$
\frac{\partial}{\partial t} \rho+\frac{2}{\left(\gamma_{\mathrm{v}}+1\right)} \nabla \cdot\left[\rho\left(\gamma_{\mathrm{v}} \overrightarrow{\mathrm{v}}\right)\right]=0 \text {. }
$$

As it should be expected, at non-relativistic speeds, when $\gamma_{\mathrm{v}} \approx 1$ because $\mathrm{v}^{2}<<c^{2}$, Eq. (14) coincides with the Madelung's continuity equation [2, 6-7]. Eq. (14) was obtained from Eq. (8) by identifying $m \rho$ with the density of a fluid extending through space. Likewise, as it was done by Madelung [2, 6-7], by identifying the velocity field of this fluid with the velocity field given by Eq. (9), we can obtain from Eq. (7) the following equation:

$$
\frac{\partial}{\partial t}\left(\gamma_{\mathrm{v}} \overrightarrow{\mathrm{v}}\right)+\nabla\left[\frac{\gamma_{\mathrm{v}}^{2}}{\left(\gamma_{\mathrm{v}}+1\right)} \overrightarrow{\mathrm{v}} \cdot \overrightarrow{\mathrm{v}}\right]+\frac{\nabla(V+Q)}{m}=0, Q=-\frac{\hbar^{2}}{\left(\gamma_{\mathrm{v}}+1\right) m} \frac{\nabla^{2} \sqrt{\rho}}{\sqrt{\rho}}
$$

If $v$ and thus $\gamma_{\mathrm{v}}$ only depend on time but not on position, Eq. (15) can be simplified in the following Euler-like equation:

$\frac{\partial}{\partial t}\left(\gamma_{\mathrm{v}} \overrightarrow{\mathrm{V}}\right)+\frac{2}{\left(\gamma_{\mathrm{v}}+1\right)}\left[\left(\gamma_{\mathrm{v}} \overrightarrow{\mathrm{v}}\right) \cdot \nabla\left(\gamma_{\mathrm{v}} \overrightarrow{\mathrm{v}}\right)\right]=-\frac{\nabla(V+Q)}{m}$.

As it should be expected, at non-relativistic speeds, when $\gamma_{\mathrm{v}} \approx 1$, Eq. (16) coincides with the Euler-like equation obtained by Madelung [2, 6-7].

\section{Example 1: plane waves}

The fluid dynamic of a classical ideal fluid flow supposes the fluid is non-viscous; the flow is steady, i.e., the velocity is time independent; the fluid is incompressible, i. e., the liquid density is constant; and also assumes that the flow is irrotational [17]. The dynamic of an ideal fluid with density $\rho$ ', which is flowing close to the Earth's surface under the influence of the 
Earth gravitational potential, $U_{g} / m=g H$, where $g$ is the gravitational acceleration and $H$ is the high respect to the ocean's surface, is given by the Bernoulli equation [17]:

$$
\frac{1}{2} \rho^{\prime} v^{2}+\rho^{\prime} \frac{U_{g}}{m}+P=\text { constant } .
$$

In Eq. (17), $P$ is the pressure inside of the liquid. While Eq. (17) is purely classical and has no connection with Madelung fluids, it is instructive to compare the Madelung liquid, associate to a free particle "guided" by a plane wave, to a classical ideal liquid under non-gravity conditions, which dynamics is described by the Bernoulli equation with $U_{g}=0$. A simple solution of the GdeP equation for a free particle $(V=0)$ is the plane wave, normalized in a large cube of side $\mathrm{L}$, given by the following equation $[11,13]$ :

$$
\psi=\frac{1}{\sqrt{L^{3}}} e^{\frac{i}{\hbar}\left(\vec{p} \cdot \vec{r}-\frac{p^{2}}{\left(\gamma_{\mathrm{v}}+1\right) m} t\right)} .
$$

In Eq. (18), $p$ is the magnitude of the particle's linear momentum, which can take any positive real value. Evaluating Eq. (18) for $\gamma_{v}=1$ gives the correct normalized plane wave when the free particle is traveling at non-relativistic speeds [6]. The surfaces of constant phase corresponding to Eq. (18) are planes perpendicular to the particle's linear momentum. Note that for a given value of $p$, the value of $\gamma_{\mathrm{v}}$ get univocally determined by the equality of the following formulas for the relativistic kinetic energy $[11,13,15]$ :

$$
K=\left(\gamma_{\mathrm{v}}-1\right) m c^{2}=\frac{p^{2}}{\left(\gamma_{\mathrm{v}}+1\right) m} \Rightarrow \gamma_{\mathrm{v}}=\frac{\sqrt{p^{2}+m^{2} c^{2}}}{m c}
$$

Using Eqs. (6), (13) and (18), we can obtain:

$$
R=\sqrt{\rho}=L^{-\frac{3}{2}} \Rightarrow Q \equiv 0, S(\vec{r}, t)=\left(\vec{p} \cdot \vec{r}-\frac{p^{2}}{\left(\gamma_{\mathrm{v}}+1\right) m} t\right) .
$$

From Eq. (20) follows that the Madelung fluid associated to a free particle guided by a plane wave has constant density $\rho^{\prime}=m \rho$; therefore, it is incompressible. It is also nonviscous because the total force acting on it is $F=-\nabla(V+Q) / m=0$. The velocity of this fluid and the corresponding value of $\gamma_{\mathrm{v}}$ can be obtained using Eqs. (10) and (20):

$$
\overrightarrow{\mathrm{v}}=\frac{c}{\sqrt{(m c)^{2}+p^{2}}} \vec{p}, \gamma_{\mathrm{v}}=\frac{\sqrt{(m c)^{2}+p^{2}}}{m c}
$$

The value of $\gamma_{v}$ given by Eqs. (19) and (21) are identical in this case, but as it will be shown in the next Section, this is not a general feature of the theory. The maximum possible value of the fluid speed, $\mathrm{v} \approx c$, occurs when $\nabla S=p \gg m c$. This corresponds to $\gamma_{\mathrm{v}} \gg 1$. The fluid velocity is constant; thus, this Madelung fluid is irrotational. Eq. (16) reduces now to:

$$
\nabla\left[\frac{\left(\gamma_{\mathrm{v}} \mathrm{v}\right)^{2}}{\left(\gamma_{\mathrm{v}}+1\right)}\right]=\frac{\nabla\left[\frac{p^{2}}{\left(\gamma_{\mathrm{v}}+1\right) m}\right]}{m}=0 \Rightarrow K=\text { constant }
$$

Evidently, Eqs. (21) and (22) also gives the correct results at the non-relativistic limit. A comparison between Eq. (22), evaluated for $\gamma_{v}=1$, and the Bernoulli equation (Eq. (17) with $U g=0$ ) shows that there is not pressure in the Madelung fluid associated to a free quantum 
particle guided by a plane wave. From Eqs. (11), (12), and (21) follow that the Bohmian paths of a free quantum particle associated to a plane wave are given by the following equation [6]:

$$
\vec{r}_{p}(t)=\vec{r}_{o}+\frac{c}{\sqrt{(m c)^{2}+p^{2}}} \vec{p} t
$$

Evidently, Eq. (23) also gives the correct result for a particle moving at non-relativistic speeds. In Eq. (23), the initial position of the particle lies everywhere in space. Like for free classical particles moving at non-relativistic speeds, these Bohmian paths are therefore uniform, rectilinear, and perpendicular to the planes of constant phase of the wave. This is because in this case the quantum potential is null, thus $\nabla Q=0$ [6]. Also note that Eqs. (14) to (16) are fulfilled because both $\rho$ and $\mathrm{v}$ are constant.

\section{Example 2: standing waves}

A simple but interesting case, where $Q$ is not null, occurs when a free quantum particle is in the superposition state formed by two plane waves, which are both solutions of Eq. (1) with $V$ $=0$ but are traveling in opposite directions along the $\mathrm{x}$-axis with the same value of $p$ :

$$
\psi(x, t)=\frac{1}{\sqrt{2 L^{3}}}\left[e^{i\left(k x-w_{k} t\right)}+e^{i\left(-k x-w_{k} t\right)}\right]=\frac{2}{\sqrt{2 L^{3}}} \cos (k x) e^{-i w_{k} t}, k=\frac{p}{\hbar}, w_{k}=\frac{p^{2}}{\left(\gamma_{\mathrm{v}}+1\right) m \hbar} .
$$

The speeds of the Madelung fluids associated to either one of these two plane waves are the same and given by Eq. (23), but the corresponding velocities point to opposite directions; therefore, $\gamma_{\mathrm{v}}$ is also the same for each wave when individually considered. Consequently, the standing wave given by Eq. (24) is also a solution of Eq. (1) with $V \equiv 0$, and with the same value of $\gamma_{\mathrm{v}}$ than for each of the plane waves components. For the standing wave:

$$
R(x)=\sqrt{\rho(x)}=\frac{2}{\sqrt{2 L^{3}}} \cos (k x) \Rightarrow Q=-\frac{\hbar^{2}}{\left(\gamma_{\mathrm{v}}+1\right) m} \frac{\frac{d^{2}}{d x^{2}} R(x)}{R(x)}=\frac{\hbar^{2} k^{2}}{\left(\gamma_{\mathrm{v}}+1\right) m}=\frac{p^{2}}{\left(\gamma_{\mathrm{v}}+1\right) m}, \quad S(t)=-\frac{p^{2}}{\left(\gamma_{\mathrm{v}}+1\right) m} t .
$$

From Eqs. (24) and (25) follows that the period of the $\cos ^{2}(k x)$ density distribution is inverse proportional to $p$. The Madelung fluid associated to a free particle guided by a standing wave does not have a constant density; therefore, it is compressible, thus, it does not behave like a classical ideal fluid flow. The wavelength of the standing wave, $\lambda$, is inverse proportional to $p$. From Eq. (25) also follows that $\nabla S=0$; therefore, from Eq. (10) follows that the velocity of this fluid is zero and $\gamma_{\mathrm{v}}=1$, which is different than the $\gamma_{\mathrm{v}}$ value corresponding to each superposing plane wave. Consequently, the Bohmian particle associated to a standing wave is at rest. In Eq. (25), $Q$ is equal to the relativistic kinetic energy of the free particle, which is constant; therefore, $\nabla Q=0$, thus this Madelung fluid is non-viscous. Eqs. (14) to (16) are now fulfilled because $\rho$ does not depend on time and $\mathrm{v}=0$. A comparison of the results obtained in this example for a standing wave, to the results obtained in the previous Section for a plane wave, illustrates the well-known nonlocality properties of the theories resulting from the Madelung-de-Broglie-Bohm reformulation of the Schrödinger equation. The superposition of plane waves, which are solutions of the same GdeP equation for a free particle, modifies the properties of the corresponding Madelung fluid, and then the Bohmian trajectories of the guided particle.

\section{Example 3: quasi-standing waves}


In this Section we will consider a wavefunction of Eq. (1) with $V=0$, which is a slightly variation of Eq. (24):

$$
\psi(x, t)=\frac{1}{\sqrt{2 L^{3}}}\left[e^{i\left(k x-w_{k} t\right)}+e^{i\left(-k^{\prime} x-w_{k^{\prime}} t\right)}\right], k^{\prime}=\frac{p+\Delta p}{\hbar}, w_{k^{\prime}}=\frac{(p+\Delta p)^{2}}{\left(\gamma_{\mathrm{v}}+1\right) m \hbar}, \Delta p \ll p .
$$

In Eq. (26), $k$ and $w_{k}$ are given by Eq. (24). Consequently, the first term of the wavefunction in Eq. (26) is a solution of Eq. (1) with $V=0$. However, the second term is not because there is, in the denominator of $w_{k}$, the same value of $\gamma_{v}$ than for $w_{k}$. From Eq. (19) follows that the value of $\gamma_{\mathrm{v}}$ corresponding to $(p+\Delta p)$ is approximately equal to the value corresponding to $p$ in two situations. First, $\gamma_{\mathrm{v}} \approx 1$ at the non-relativistic limit when $p \ll m c$. Second, $\gamma_{\mathrm{v}} \approx p / m c$ at the ultra-relativistic limit $p \gg m c$; therefore, $(p+\Delta p) / m c \approx p / m c$ when $\Delta p<<m c$. Consequently, at these two limits the second term of Eq. (26) is approximately a solution of Eq. (1) with $V=$ 0 . We will call here, a quasi-standing wave, to the wavefunction given by Eq. (26) at these two limits. After some straightforward algebraic steps for transforming Eq. (26) in a form like Eq. (6), we obtained the following results:

$$
\begin{aligned}
& \nabla \mathrm{S}=\frac{-\Delta p}{2} \hat{x} \Rightarrow \mathrm{v}=\frac{-c \Delta p}{\sqrt{4 m^{2} c^{2}+(\Delta p)^{2}}} \Rightarrow \gamma_{\mathrm{v}}=\frac{1}{\sqrt{1-\frac{(\Delta p)^{2}}{4 m^{2} c^{2}+(\Delta p)^{2}}}} . \\
& \rho(x, t)=\frac{2}{L^{3}} \cos ^{2}\left[k_{q}\left(x+\mathrm{v}_{q} t\right)\right], k_{q}=\frac{2 p+\Delta p}{2 \hbar}, \mathrm{v}_{q}=\frac{\Delta p}{\left(\gamma_{\mathrm{v}}+1\right) m} \Rightarrow Q=\frac{(2 p+\Delta p)^{2}}{4\left(\gamma_{V}+1\right) m} .
\end{aligned}
$$

As it should be expected, Eqs. (27) and (28) reduces to Eqs. (25) when $\Delta p=0$. The density of the Madelung fluid associated to a free particle guided by a quasi-standing wave resembles a "standing wave" that is drifting without dispersion, in the direction of the plane wave associated with the linear momentum $p+\Delta p$, with speed $\mathrm{v}_{q} \approx \mathrm{v} \approx \Delta p / 2 m$ when $\Delta p<r c$. It can be easily checked out that the quasi-standing wave given by Eq. (26) satisfies Eq. (1) with $V=0$, and with $\gamma_{v}$ given by Eq. (27). From Eqs. (27) and (28) also follows that Eqs. (7), (8), and (14) to (16) are satisfied. Note that $\gamma_{\mathrm{v}} \approx 1$ for every $p$; consequently, the Bohmian particle associated to a quasi-standing wave moves like a classical particle even at the ultra-relativistic limit.

\section{Example 4: beats}

In this Section we will consider another wavefunction of Eq. (1) with $V=0$, which can be obtained from Eq. (26) after substituting $-k^{\prime}$ by $+k^{\prime}$. Eq. (26) corresponds to the superposition of two plane waves with slightly different values of $p$ traveling in opposite directions. Here we will consider what happens when the two waves travel in the same direction. In this case, we obtained the following results:

$$
\begin{aligned}
& \nabla \mathrm{S}=p+\frac{\Delta p}{2} \hat{x} \Rightarrow \mathrm{v}=\frac{c(2 p+\Delta p)}{\sqrt{4 m^{2} c^{2}+(2 p+\Delta p)^{2}}} \Rightarrow \gamma_{\mathrm{v}}=\frac{1}{2 \sqrt{\frac{m^{2} c^{2}}{4 m^{2} c^{2}+(2 p+\Delta p)^{2}}}} . \\
& \rho(x, t)=\frac{2}{L^{3}} \cos ^{2}\left[k_{b}\left(x-\mathrm{v}_{b} t\right)\right], k_{b}=\frac{\Delta p}{2 \hbar}, \mathrm{v}_{b}=2 \frac{\frac{1}{2}[p+(p+\Delta p)]}{\left(\gamma_{\mathrm{v}}+1\right) m} \Rightarrow Q=\frac{(\Delta p)^{2}}{4\left(\gamma_{V}+1\right) m} .
\end{aligned}
$$

Note that $k_{b}$ does not depend on $p$ but is proportional to $\Delta p$. Therefore, as it should be expected, Eqs. (29) and (30) reduce when $\Delta p=0$ to Eqs. (20) and (21), which correspond to the 
first example of a single plane wave discussed in Section IV. The Madelung fluid now flows without dispersion in the same direction than the plane waves, and at the average speed of both waves. The factor of 2 at the front of Eq. (30) for $\mathrm{v}_{b}$ is because a $\cos ^{2}(a y-b t)$ shaped wave travels at twice the speed than a $\cos (a y-b t)$ shaped one. Consequently, the corresponding Bohmian paths are uniform and rectilinear at both non-relativistic and relativistic values of $\mathrm{v}_{b}$. This result suggests the following very interesting possibility: a free relativistic quantum particle could be associated to a Gaussian pulse, which is formed by a superposition of plane waves traveling in the same direction with similar values of $p$, and thus could be a solution of Eq. (1) with $V=0$. Such a Gaussian pulse would travel with no dispersion at the average relativistic speed of all the plane waves forming the Gaussian pulse.

\section{Conclusions}

Madelung, de Broglie, and Bohm reformulated the Schrödinger equation and founded the non-relativistic quantum hydrodynamics and quantum mechanics with trajectories. Following a similar procedure, we reformulated the GdeP equation for extending quantum hydrodynamics and quantum mechanics with trajectories to the relativistic domain. As it should be expected, we showed that at non-relativistic energies, the resulting equations coincide with the well-known non-relativistic equations. As a prove of the potential practical value of the formulated theory, we found the relativistic solutions of some simple but instructive free particle problems.

\section{References}

1. Schrodinger E., Quantisierung als Eigenwertproblem. I and II, Ann. Phys. 79 361-376, 489-527 (1926).

2. Madelung E. and Zeit. F. Quantentheorie in hydrodynamischer form, Phys. 40 322-326 (1927).

3. Landau L.D. and Lifshitz E.M., Fluid Mechanics, (Pergamon Press, 1987).

4. Bohm D., A Suggested Interpretation of the Quantum Theory in Terms of "Hidden" Variables, Phys. Rev. 85 166-179 (1952).

5. Bohm D., Comments on a Letter Concerning the Causal Interpretation of the Quantum Theory, Phys. Rev. 89319 (1953).

6. Holland P. R., The quantum theory of motion: an account of the de Broglie-Bohm causal interpretation of quantum mechanics (Cambridge University Press, Cambridge, 1993).

7. Wyatt R. E., Quantum dynamics with trajectories: introduction to quantum hydrodynamics (Springer, New York, 2005).

8. de Broglie L., A Tentative Theory of Light Quanta, Phil. Mag. 47 446-458 (1924).

9. Poirier B., Trajectory-based Theory of Relativistic Quantum Particles, J. Physics, 701, 012013 (2016).

10. Struyve W. and Valentine A., De Broglie-Bohm Guidance Equations for Arbitrary Hamiltonians, J. Phys. A: Math. Theor. 42, 035301 (2008).

11. Grave de Peralta L., Natural extension of the Schrödinger equation to quasi-relativistic speeds, J. of Modern Phys. 11, 196-213 (2020).

12. Grave de Peralta L., Quasi-relativistic description of Hydrogen-like atoms, J. of Modern Phys. 11, 788-802 (2020).

13. Grave de Peralta L., Quasi-relativistic description of a quantum particle moving through one-dimensional piecewise constant potentials, Results in Phys. 18, 103318 (2020).

14. Grave de Peralta L., Exact quasi-relativistic wave functions for Hydrogen-like atoms, Scientific Reports 10, 14925 (2020). 
15. Grave de Peralta L., Did Schrödinger have other options? European J. of Phys. 41, 065404 (2020).

16. Christodeulides C., The Special Theory of Relativity (Springer, New York, 2016).

17. Walker J., Halliday D. and Resnick R., Fundamentals of Physics (10th ed., Wiley, USA, 2014).

\section{Competing interest}

The authors declare no competing interests.

\section{Additional information}

Correspondence and requests for materials should be addressed to the corresponding author. 